\title{
Coronary Artery-Left Ventricular Fistula with Giant Right Coronary Aneurysm: A Case Report and Literature Review
}

\author{
Wenjie Diao, MD,${ }^{1,2}$ Chao Shi, MD, $\mathrm{PhD},{ }^{1}$ Ge Liu, $M D,{ }^{1}$ Xuegang Liu, $M D, \mathrm{PhD}^{1,2}$ \\ ${ }^{1}$ Department of Cardiac Surgery, The First Affiliated Hospital of Bengbu Medical College, Bengbu, Anhui, P.R. China; \\ ${ }^{2}$ Shandong University, Jinan, Shandong, China
}

\section{ABSTRACT}

Right coronary artery-left ventricular (RCA-LV) fistula with associated giant right coronary artery aneurysm (CAA) is an extremely rare cardiac condition. This case study presents a patient with a large left ventricle (LV) and a giant right CAA with a maximal inner diameter of approximately $56.6 \mathrm{~mm}$ and an inner diameter of approximately $22 \mathrm{~mm}$ at its communication with the left ventricle. The patient underwent surgical management, involving suturing of the proximal end of the CAA and coronary artery bypass grafting (CABG). RCA-LV fistula with a giant right CAA may involve serious complications, such as thrombosis, rupture, and heart failure. Therefore, it is necessary to establish effective management strategies for this condition. Although this case is not unique, it serves as an illustrative example of the implementation of a classic surgical treatment method.

\section{INTRODUCTION}

Both congenital coronary artery fistulae (CAF) and giant CAAs are exceedingly uncommon anatomical abnormalities [Vavuranakis 1995], with CAAs having a prevalence of $0.02 \%$ [Gottesfeld 2004]. RCA-LV fistula with a giant right CAA is rarely observed in clinical practice. This study reported a patient whose right coronary artery (RCA) was wholly dilated, and who underwent surgical management with good prognosis. Fifteen previously reported cases of giant right CAA associated with coronary fistulae also were reviewed [Shizukuda 1989; Nakamura 1998; Li 2005; Wei 2011; Zheng 2012; Jiang 2014; Sarasa 2015; Li 2015; Detorakis 2015; Zhu 2016; Li 2017; Uzuka 2018; Sun 2019].

\section{CASE REPORT}

A 48-year-old male was admitted to the hospital with a one-year history of heart palpitations and chest tightness. The patient's past medical history included hypertension, and his

Received March 7, 2021; accepted March 25, 2021.

Correspondence: Xuegang Liu, Department of Cardiac Surgery, The First Affiliated Hospital of Bengbu Medical College, Bengbu 233004, Anbui, P.R. China (e-mail:xgliu999@126.com). physical examination revealed a continuous murmur on aortic valve auscultation. Electrocardiogram (ECG) showed premature atrial and ventricular contractions, chest X-ray showed a significant expansion on the right-heart and left-heart border,

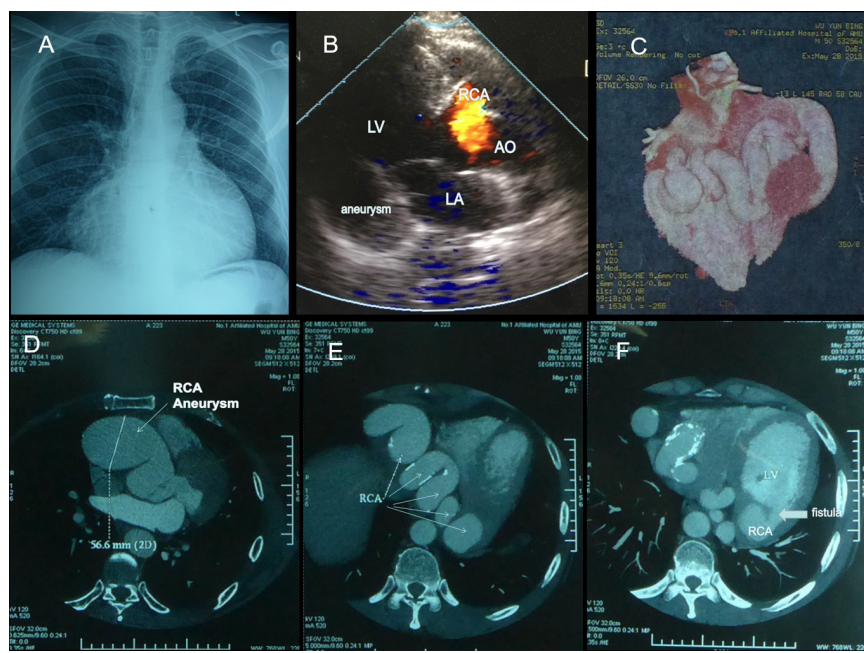

Figure 1. A, A Chest X-ray showed a significant expansion on the right-heart and left -heart border; B, Transthoracic echocardiography revealed a huge right coronary artery aneurysm; C, Three-dimensional computed tomography (CT) showing the coronary artery aneurysm (CAA); C-F) A CT scan showing the CAA being wholly dilated and a fistula at the left ventricle (LV). RCA, right coronary artery; LV, left ventricle

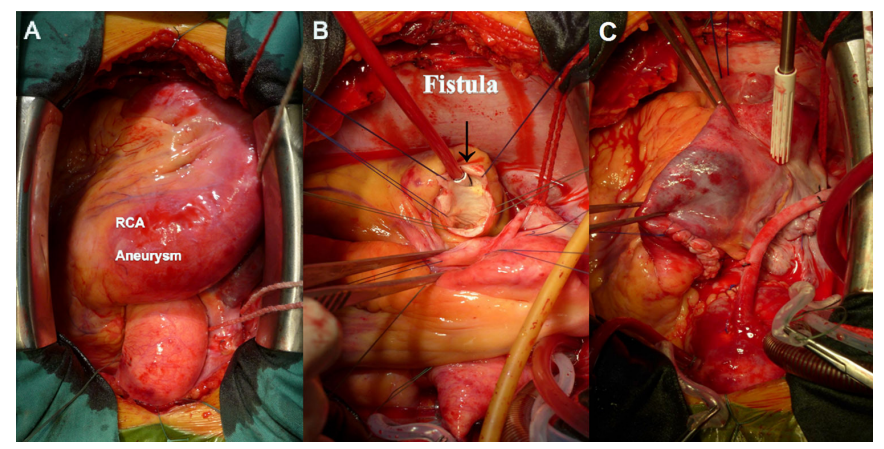

Figure 2. A, Aneurysmal sac, right coronary artery (RCA); B, RCA ostium as demonstrated by the arrow; $\mathrm{C}$, Closure of the beginning of RCA ostium with a vein graft to the distal RCA. 
Reported cases of right coronary artery-left ventricular fistula with giant right coronary aneurysm

\begin{tabular}{|c|c|c|c|c|c|c|c|c|}
\hline \multirow[t]{2}{*}{ Nakamura $Y$ et al. } & 1998 & $57 / M$ & Chest oppression & RCA-LV & 80 & 15 & NA & CARC, closure \\
\hline & 2005 & - & Palpitation, fatigue & RCA-LV & $138 * 121 * 110$ & 22 & $\mathrm{Al}$ & $C A B G * 2, A V R$, closure \\
\hline Li et al. & 2005 & - & Angina, chest pain & RCA-LV & $56 * 54 * 52$ & NA & $\begin{array}{c}\text { Aortic dilation, Al, } \\
\text { thrombus }\end{array}$ & $\begin{array}{l}\text { CARC, AVR, AP, } \\
\text { closure }\end{array}$ \\
\hline Wei et al. & 2011 & $26 / M$ & Asymptomatic & RCA-LV & 58 & NA & NA & $C A B G * 1$, closure \\
\hline Zheng et al. & 2012 & $47 / M$ & $\begin{array}{c}\text { Chest distress, body } \\
\text { weakness }\end{array}$ & RCA-LV & 70 & 20 & Thrombus & CARC, closure \\
\hline Li et al. & 2015 & $51 / M$ & Cough, white sputum & RCA-LV & $120 * 110$ & 18 & NA & CABG, closure \\
\hline Detorakis et al. & 2015 & $42 / M$ & Asymptomatic & RCA-LV & 56 & NA & NA & CABG, closure \\
\hline Zhu et al. & 2016 & $45 / M$ & Chest congestion & RCA-LV & $100 * 61$ & 8 & RVH & CABG, closure \\
\hline Li et al. & 2017 & $38 / M$ & $\begin{array}{l}\text { Chest distress, } \\
\text { palpitations }\end{array}$ & RCA-LV & 21 & NA & NA & Closure \\
\hline Uzuka et al. & 2018 & $64 / F$ & Palpitations & RCA-LV & 60 & 4 & Thrombus & $\begin{array}{c}\text { CABG*1, } \\
\text { Embolectomy, closure }\end{array}$ \\
\hline Sun et al. & 2019 & $55 / M$ & Chest pressure & RCA-LV & 50 & NA & NA & CARC, closure \\
\hline Ours & 2020 & $48 / M$ & $\begin{array}{l}\text { Chest distress, palpita- } \\
\text { tions }\end{array}$ & RCA-LV & $77 * 55$ & 22 & NA & $C A B G * 1$, closure \\
\hline
\end{tabular}

CAA, coronary artery aneurysm; RCA, right coronary artery; LV, left ventricle; CARC, coronary artery reconstruction; Al, aortic valve insufficiency; a: Mean 30-56 years; CABG, coronary artery bypass grafting; AVR, aortic valve replacement; SB, shortness of breath; AP, aortoplasty; MI, mitral regurgitation; MVP, mitral valvuloplasty; NVCM, noncompaction of the ventricular myocardium; RVH, right ventricular hypoplasia

while transthoracic echocardiogram and three-dimensional computed tomography (CT) demonstrated a giant right CAA with a maximal inner diameter of about $56.6 \mathrm{~mm}$, with its terminal communication with the left ventricle measuring approximately $22 \mathrm{~mm}$ in diameter (Figure 1).

The patient underwent surgical management with cardiopulmonary bypass, with an arterial cannula inserted into the femoral artery and an intravenous cannula inserted into the femoral vein. The arrested heart then underwent direct perfusion through the coronary artery sinus and retrograde perfusion through the coronary venous sinus. Intraoperatively, the heart was found to be hypertrophic, and the RCA was wholly dilated and locally calcified with an opening measuring approximately $4.0 \mathrm{~cm}$. The diameter and length of the right CAA was approximately $5.5 \mathrm{~cm}$ and $20 \mathrm{~cm}$, respectively. Regarding the left heart, the diameter of the $\mathrm{LV}$ fistula was about $22 \mathrm{~mm}$ and the left coronary artery was slightly thicker (Figure 2A, B). The aortic valve was probed, and no regurgitation was found. A continuous 5-0 polypropylene line was used to suture the LV septum and RCA opening. The left saphenous vein bypassed between the formed distal aneurysm and the ascending aorta, and a right coronary aneurysmoplasty was performed by making a tunnel between the right CAA residual cavity and the right atrium (Figure 2C). The operative time was approximately five hours, and the intraoperative blood loss was around $400 \mathrm{ml}$.

The patient recovered well and was discharged on the 14th postoperative day without complications.

\section{DISCUSSION}

Most coronary artery fistulae are congenital and usually involve the right heart, left heart, and pulmonary artery. The causes of CAA included coronary atherosclerosis, Kawasaki disease, congenital coronary dysplasia, trauma, and arterial dilatation after interventional therapy [Kamiya 2002]. Giant CAA in adults is defined as having a diameter greater than $20 \mathrm{~mm}$.

$\mathrm{RCA}-\mathrm{LV}$ fistulae with giant right CAAs are rare. A literature search on the PubMed database for case reports between 
1989 and 2020, using the keyword "giant right coronary artery aneurysm, left ventricular fistula," yielded 15 cases (Table 1). Of the 16 total RCA-LV cases (including this report), three were female, nine were male, and four cases did not disclose sex. The diameter of the CAAs ranged from $21 \mathrm{~mm}$ to $110 \mathrm{~mm}$. Most patients had no obvious symptoms in the early stages; however, as the disease progressed, the most commonly observed signs and symptoms were angina, myocardial infarction, arrhythmia and palpitation, shortness of breath, fatigue, chest tightness, and cough. The size of the fistulae ranged from $4 \mathrm{~mm}$ to $22 \mathrm{~mm}$, and fistulae most commonly were located in the posterior and inferior walls of the left ventricle; the fistula in our case was located in the posterior wall of the left ventricle. Due to the slow blood flow in aneurysms, CAAs may be associated with thrombosis; accordingly, thrombosis was reported in four cases [Li 2005; Zheng 2012; Uzuka 2018]. Huge aneurysm can compress the heart and cause dysplasia, and this was observed with one case of right ventricular hypoplasia (RVH) [Zhu 2016], one case of noncompaction of the ventricular myocardium (NVCM) [Jiang 2014], and other various cardiac changes, including aortic dilatation, aortic valve insufficiency [Li 2005], and mitral regurgitation [Sarasa 2015]. In our case study, large amounts of blood flowed from the RCA into the LV, causing LV hypertrophy and dysfunction; however, the clinical manifestations may vary across patients, due to the size of the CAA and location of the fistula.

Coronary angiography is typically the best choice for assessing CAA; however, multi-slice spiral CT coronary angiography may provide additional information regarding the number, size, and shape of CAAs [Schmid 2006]. In addition, it could reveal the extraluminal components, such as thrombosis and aneurysm shape, which cannot be assessed by coronary angiography. Echocardiography and magnetic resonance imaging also have diagnostic value [Liu 2019].

Giant CAAs have many fatal complications, including thrombosis, rupture, myocardial ischemia, and LV dysfunction [Sugiyama 2017; Ahmed 1982]. Therefore, surgical management should occur as soon as possible after diagnosis [Matsumoto 2018].

A femoral artery cannula was used during surgery, due to the aneurysm's size and location, which precluded the use of an ascending aorta cannula. The heart was operated on by direct perfusion through the coronary artery sinus and retrograde perfusion through the coronary venous sinus. Using the preoperative findings, the fistula could accurately be located and was repaired using a horizontal mattress suture. After exploring the aortic valve, no regurgitation was encountered. A continuous 5-0 polypropylene line was then used to suture the LV septum and RCA opening. The left saphenous vein bypassed between the formed distal aneurysm and the ascending aorta. A tunnel between the right CAA residual cavity and right atrium was made to shunt the residual blood. The ECG confirmed that no myocardial ischemia occurred during or after the operation. The patient recovered well postoperatively, and there were no complications during the 5 -year follow up.

Given the limited experience clinicians may have with the treatment of RCA-LV fistula with giant right CAA and the seriousness of its related complications, we completed this report to further inform the comprehensive diagnosis and treatment of this conditions.

\section{ACKNOWLEDGEMENT}

This work was supported by grants from Anhui Science and Technology Project (1804h08020280). The authors wish to thank Drs. Xiaohong Li and Dr. Yiyao Jiang for their assistance in the cardiopulmonary bypass procedure.

\section{REFERENCES}

Ahmed SS, Haider B, Regan TJ. 1982. Silent left coronary artery-cameral fistula: probable cause of myocardial ischemia. Am Heart J. 104: 869-70.

Detorakis EE, Foukarakis E, Karavolias G, Dermitzakis A. Dermitzakis A. 2015. Cardiovascular magnetic resonance and computed tomography in the evaluation of aneurysmal coronary-cameral fistula. J Radiol Case Rep. 9, 10-21.

Gottesfeld S, Makaryus AN, Singh B, et al. 2004. Thrombosed right coronary artery aneurysm presenting as a myocardial mass. J Am Soc Echocardiogr. 17: 1319-22.

Jiang B, Yang Y, Li F, et al. 2014. Giant Aneurysm of Right Coronary Artery Fistula Into Left Ventricle Coexisting With Noncompaction of Left Ventricular Myocardium. Ann Thorac Surg. 98 (4): e85-6.

Kamiya H, Yasuda T, Nagamine H, et al. 2002. Surgical treatment of congenital coronary artery fistulas: 27 years experience and a review of the literature. J Card Surg. 17: 173-7.

Li D, Wang Y, An Q. 2017. An aneurysmal right coronary artery fistula draining into the left ventricle. Cardiol Young. 27: 1214-15.

Li D, Wu Q, Sun L, et al. 2005. Surgical treatment of giant coronary artery aneurysm. J Thorac Cardiovasc Surg. 130: 817-21.

Li Y, Xie M, Wang X, Lv Q, Yang Y, He L. 2015. Two Giant Right Coronary Artery Aneurysms with Fistula to the Left Ventricle: Preliminary Diagnosis by Echocardiography. Echocardiography. 32 (6): 1053-5.

Liu J, Wang X, Sun D, Yang J. 2019. Dual Aneurysms of the Left Anterior Descending Coronary Artery Initially Detected by Echocardiography. Int Heart J. 60 (3): 778-83.

Matsumoto Y, Kawano H, Iwasaki K, Arakawa S, Maemura K. 2018. Histopathology of Giant Coronary Artery Aneurysm Associated with Coronary Artery Fistula. Int Heart J. 59 (2): 431-4.

Nakamura Y, Yutani C, Imakita M, et al. 1998. A huge coronary aneurysm resulting from a coronary artery-to-left ventricle fistula. Intern Med. 37: 366-9.

Sarasa I, Daihiko H, Susumu I, Katsumi H, Takeshi A. 2015. Right coronary artery--left ventricle fistula with giant coronary artery aneurysm, European Heart Journal-Cardiovascular Imaging. 16 (2): 231.

Schmid M, Achenbach S, Ludwig J, et al. 2006. Visualization of coronary artery anomalies by contrast-enhanced multi-detector row spiral computed tomography. Int J Cardio. 111: 430-5.

Shizukuda Y, Yonekura S, Tsuchihashi K, Tanaka S, Komatsu S, Iimura O. 1989. A case of a right coronary artery to left ventricle fistula observed over twenty years. Jpn J Med. 28 (4): 510-4. 
Sugiyama K, Suzuki S, Kamiya K, Koizumi N, Ogino H. et al. 2017. Ruptured coronary artery aneurysm with pulmonary artery fistulae. J Card Surg. 32: 799-800.

Sun JP, Yang L, Zhao Z, Zhang X, Ma G. 2019. A rare right coronary artery-left ventricular fistula with giant coronary artery and aneurysm. Eur Heart J Cardiovasc Imaging. 20 (5): 604.

Uzuka T, Nakamura M, Nakajima T, Watanabe N, Fukazawa Y. 2018. Surgery of giant right coronary artery aneurysm complicated with coronary artery fistula to left ventricle. J Card Surg. 33: 95-6.

Vavuranakis M, Bush CA, Boudoulas H. 1995. Coronary artery fistulas in adults: incidence, angiographic characteristics, natural history. Cathet
Cardiovasc Diagn. 35: 116-20.

Wei J, Shu Y, Wei X, et al. 2011. Right coronary artery fistula to left ventricle complicated with huge coronary artery aneurysm. Intern Med. 50: $239-42$.

Zheng J, Dong R, Liu T, Li Y, Zhou Q. 2012. Giant right coronary artery aneurysm with a fistula to the left ventricle. Ann Thorac Surg. 94: e149-50.

Zhu F, Zheng Z, Yao L, Mou Y, Cheng Y, Gao H. 2016. Isolated right ventricular hypoplasia caused by giant aneurysm of right coronary artery to left ventricle fistula in an adult: a case report. J Card Surg. 11 (1): 93. 